\title{
Evaluation of Reading Skill in Children with Developmental Coordination Disorder in Indian Context
}

\author{
Ganapathy Sankar $\mathbf{U}^{1 *}$ and Monisha $\mathbf{R}^{2}$ \\ ${ }^{1}$ Department of Occupational Therapy, SRM Institute of Science and technology, India \\ ${ }^{2}$ Department of Physiotherapy, SRM Institute of Science and Technology, India \\ *Corresponding author: Ganapathy Sankar U, Department of Occupational Therapy, SRM Institute of Science and technology, India
}

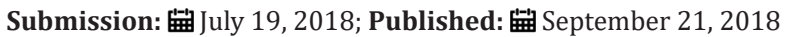

\section{Introduction}

Children with Impairment in motor coordination and without any neurological or pervasive developmental disorder has been identified as Developmental Coordination Disorder, The DSM IV coined the term developmental coordination disorder (DCD) to identify children who were previously identified by the parents and teachers as CLUMSY. These children have impairment in motor coordination that makes activities of daily living difficult and contributes to poor academic achievement [1]. Researchers around 2003 were trying to identify the cause behind DCD and they believed that it is due to the deficit in the cortical system that prevents messages from being properly transmitted to the body and the result is in-coordination. But the etiology of DCD is still left out as Hypothesis [2,3]. Predominant feature reported in all children with DCD is Clumsiness. They have poor postural adaptation and when assigned a fine or gross motor activity, initially there lies confusion about which hand or leg to use first? Children with DCD avoid physical activity with their peer group in physical training session in school and at homes, due to the difficulties in throwing or catching a ball. Wide array of Visual- perceptual deficit exist in Children with DCD, they have trouble in task requiring motor control, learning and cognition. Frequently reported difficulty in these children is difficulty in following motor commands, difficulty in sequencing task and Memory. They require increase in time to complete a task as compared to the age matched peer groups. Difficulty with handwriting and learning is the predominant deficit.

If proper identification of DCD is not done, they will be passing from one service to next to achieve good result in academics and in physical activity [4]. Inability to hold a pen or pencil properly is the observable feature. Findings from longitudinal studies indicate that children with DCD is left untreated they experience difficulties throughout the adolescence Visual proprioception is impaired and it is the characteristic of children with DCD. They have difficulty in tasks like length and size differentiation. Common failures have been found in estimating object and in locating an object's position in space. These children have been reported to have Cognitive deficit. There exist reading problem and learning disabilities. However, none of the researchers have concerned towards analyzing the reading disability and learning problems in these group of children with DCD. However, very little work has done analyzing the working memory of this group. Hence the current study has done to understand the memory associated with this group of children and to explore the link between impairments of working memory and reading difficulty. Working memory should be assessed in children at the baseline as this memory is needed to execute learning, reasoning and manipulating information to achieve the task [5].

Every individual has varying capacities in their verbal memory and working memory. Verbal short-term memory is needed for academic achievement. However, researchers around 1998 have proved that Verbal short-term memory skills are weakly associated with general academic and cognitive performance than working memory. There is a specific link between verbal short-term memory and the learning new words in early childhood years, and this Verbal memory is needed in learning second language at all ages. Children with DCD have poor verbal short term memory and they have deficit in the process of learning new words and thus shows poor academic performance. However, they acquire knowledge in new vocabulary items at a much slower rate than the peer groups. Verbal memory will effectively predict performance of children with DCD in more complex activities like Reading and in language comprehension [6]. Children with DCD have deficit in verbal working memory rather than having normal IQ were identified to have difficulty in both reading and mathematical abilities. Previous evidence has suggested that visuospatial memory supports number representation, such as place value and alignment in columns, in arithmetic. There have been very few studies that have looked at the performance of children with DCD on reading ability $[7,8]$. 


\section{Methodology}

The participants were 10 children with the age group of 8 and10 years, referred for learning difficulties especially reading to the Rehabilitation center. The whole study has been conducted for a period of 2 years (from 2016 to 2018). All the participants were diagnosed as having DCD based on the DSM-5 criteria for DCD and with reference to MABC scores. Reading speed has been assessed by the previous researchers by using Passage Reading Test. Children with emotional crisis, ADHD, LD or presence of neurological deficit and children not going to school for the past 2 years, who has been under regular handwriting training program and undergoing special classes for handwriting and drawing were excluded from the study. Initially children's dominant limb has been assessed and documented. Based on the M-ABC test result children have been divided into two groups, group A with DCD and group B with non- DCD. MABC identifies motor coordination difficulties. M-ABC identifies motor ability by measuring manual dexterity, balance skill and ball throwing and catching skill. Score can be calculated as 0 -assigned for best score and 5-assigned for poorest test performance result. By calculating raw score (0 to 40) on each item, children performance can be compared with their peer groups [9].

\section{Data analysis}

Test result can be calculated by calculating the total score in 3 subtests. If the child scores below $5^{\text {th }}$ percentile in one of the three M-ABC different sub scores, which indicate a high level of motor impairment, were included in the Group A. Children who scored above the $15^{\text {th }}$ percentile on the M-ABC total score and above the $5^{\text {th }}$ percentile in all the M-ABC sub scores were included in the group B. Visual-perceptual skills were measured using the Test of VisualMotor Integration (VMI) Is used to test the difference exist between Group A and B subjects. In VMI, motor integration and visual perception is assessed by asking the children in both the groups to copy the geometric forms within the time limits of 3 min using pencil. Scoring can be done by assigning a score of 1 for copying the exact geometric forms and score of 0 is assigned for missed items. The raw score is 30. Next each child in Group A and B have been assessed with passage reading test developed by Cornoldi and Colpo in 1981. This instrument documents the reading speed and increasing number of syllables in each passage according to grades. Grade two has 250 syllables and 570 in eighth grades. Each child has been instructed to read out loud at the beginning of the test and the number of errors made by the child in each grade has been documented in $[10,11]$.

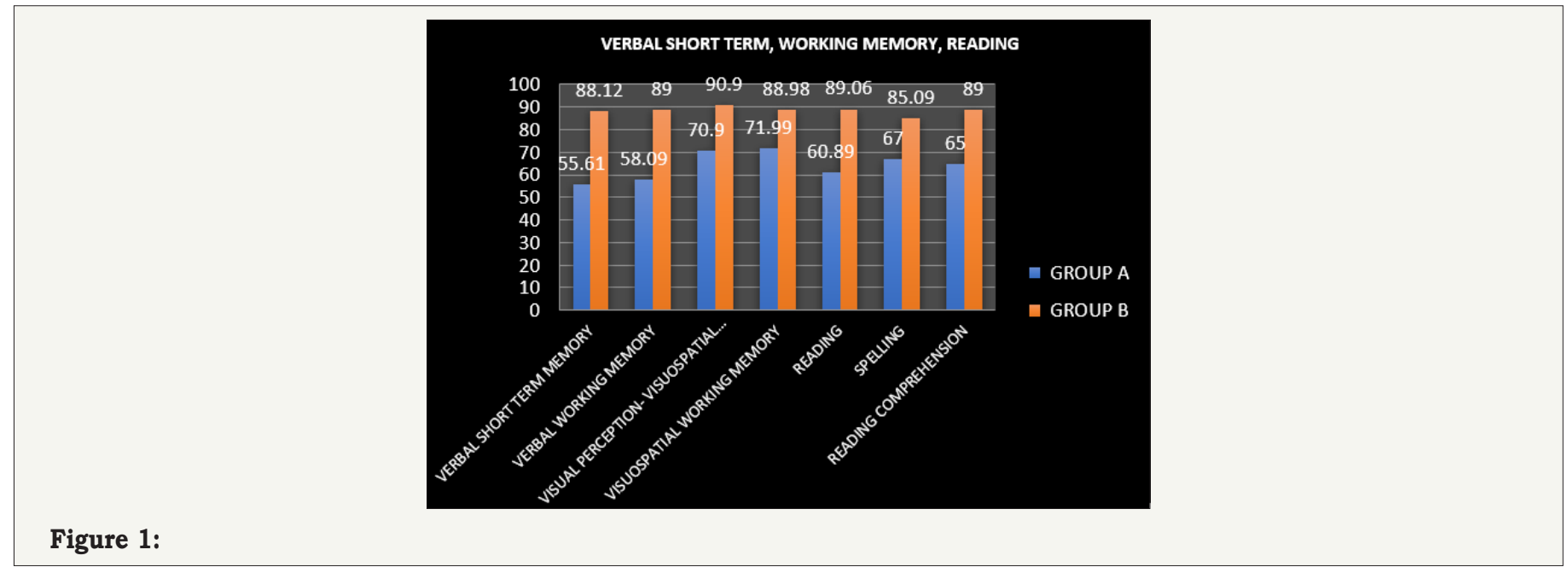

Table 1: Standard scores on verbal short term, working memory, reading.

\begin{tabular}{|c|c|c|c|c|}
\hline Measures & Mean- Group a & Mean- Group b & Sd-Group a & Sd-Group b \\
\hline Verbal short-term memory & 55.61 & 88.12 & 12 & 18.06 \\
\hline Verbal working memory & 58.09 & 89 & 13.01 & 18.56 \\
\hline Visual perception-Visuospatial short-term memory & 70.9 & 90.9 & 15.09 & 19.33 \\
\hline Visuospatial working memory & 71.99 & 88.98 & 15.18 & 18.34 \\
\hline Reading & 60.89 & 89.06 & 13.98 & 18.97 \\
\hline Spelling & 67 & 85.09 & 13.87 & 17.09 \\
\hline Reading Comprehension & 65 & 89 & 13.99 & 18 \\
\hline
\end{tabular}

Table 1, Figure 1.

\section{Discussion}

The present study provides a detailed analysis of the relation between working memory and reading ability in children with
DCD. The defect observed in visuospatial short-term and working memory were significantly poorer in group A than group B. Reading skills were poor, with greater associations between visualproprioception and Reading even after having IQ score of above 
70. They were divided based on MABC score into DCD and NonDCD. In verbal working memory skills, there was a statistically significant difference found between Group A and B. To analyze the children's visual-spatial memory they have been asked to perform Counting Recall task and Backward Digit Recall task, these children performed worse on the above mentioned tasks, which did not require movement. However, the visual-spatial memory performance was not worse than verbal working memory. It contributes to poor reading performance in passage reading test. Motor deficit in addition with visual processing deficits provide difficult for children with DCD. It has been proved that children with low visuospatial memory skills performed significantly worse in reading task as compared to Group B and the aim of the present study was the investigation of Reading ability in the population of children with DCD as compared to the Non-DCD group and it has been concluded from the test that the two subgroups have significant difference regarding reading speed. The findings of this present pilot study are not correlated with the findings of previous researchers

\section{Conclusion}

This pilot study reveals that motor impairment and visualperceptual deficit is responsible for the reading difficulty. Development of a valid tool in the identification of reading difficulty in children with DCD is in increasing need. Further experiments are needed over a larger sized sample, using other neuro psychological measures strictly connected with language-based learning disorders, such as attention and memory.

\section{Acknowledgment}

We thank all the children and families who took part in this study. No financial relationship, funding, or donation were perceived by the authors for conducting this research and writing this article.

\section{References}

1. American Psychiatric Association (2013) Diagnostic and statistical manual of mental disorders. ( $5^{\text {th }}$ edn), USA.

2. Beery KE, Beery NA (2004) Beery VMI: the beery-buktenica developmental test of visual-motor integration $\left(5^{\text {th }}\right.$ edn), Pearson, London, UK.

3. Berninger VW, Nielsen KH, Abbott RD, Wijsman E, Raskind W (2008) Writing problems in developmental dyslexia: under-recognized and under-treated. Journal of School Psychology 46(1): 1-21.

4. Biotteau M, Chaix Y, Albaret JM (2015) Procedural learning and automatization process in children with developmental coordination disorder and/or developmental dyslexia. Human Movement Science 43: 78-89.

5. Biotteau M, Péran P, Vayssière N, Tallet J, Albaret JM, et al. (2017) Neural changes associated to procedural learning and automatization process in developmental coordination disorder and/or developmental dyslexia. European Journal of Pediatric Neurology 21(2): 286-299.

6. Capellini AS, Coppede AC, Valle TR (2010) Fine motor function of school aged children with dyslexia, learning disability and learning difficulties. Pro Fono 22(3): 201-208.

7. Ganapathy SU, Saritha S (2011) A study of prevalence of developmental coordination disorder (DCD) at kattankulathur, Chennai. Indian Journal of Physiotherapy and occupational therapy 5(1): 63-65.

8. Ganapathy SU (2018) The prevalence of developmental coordination disorder at Kattupakkam, Tamilnadu. IOSR Journal of Pharmacy 8(2): 49-52.

9. Coltheart M, Rastle K, Perry C, Langdon R, Ziegler JC (2001) DRC: A dual route cascaded model of visual word recognition and reading aloud. Psychological Review 108(1): 204-256.

10. Angiulli A, Siegel LS (2003) Cognitive functioning as measured by the WISC-R: Do children with learning disabilities have distinct patterns of performance? Journal of Learning Disabilities 36(1): 48-58.

11. Das T, Padakannaia P, Pugh KR, Singh NC (2011) Neuroimaging reveals dual routes to reading in simultaneous proficient readers of two orthographies. Neuroimage 54(2): 1476-1487.
Creative Commons Attribution 4.0

International License

For possible submissions Click Here
Submit Article

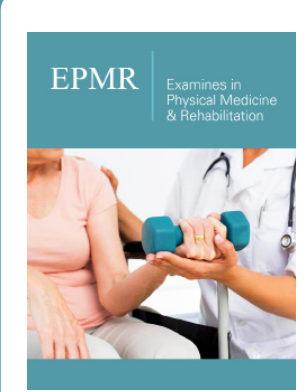

Examines in Physical Medicine and Rehabilitation: Open Access

\section{Benefits of Publishing with us}

- High-level peer review and editorial services

- Freely accessible online immediately upon publication

- Authors retain the copyright to their work

- Licensing it under a Creative Commons license

- Visibility through different online platforms 